Constructing a reliable device that is capable of processing information in a quantum mechanical way poses a formidable challenge. The logical quantum bits have to be implemented in a way that makes them sensitive to quantum mechanical effects, while at the same time this behaviour should not be disturbed by the noise of its surroundings. No matter how well designed our future quantum computers will be, they have to be able to deal with the inevitable occurrence of errors - like every classical computer ${ }^{4}$. The theory of fault-tolerant quantum computing looks at designs of quantum circuits that are able to withstand errors by storing the logical quantum bits in a redundant way, making it possible to detect and correct the errors before they affect the rest of the computation. The celebrated threshold theorem ${ }^{5}$ in quantum computing tells us that if the error rate of our components is small enough, then the benefits of using quantum error correction outweighs the overhead that is needed to do so.

Fault-tolerant computing below such a noise threshold is like a self-supporting ecosystem: although components die and garbage is produced, the dynamics of the system takes care of such disruptions and without the need for external intervention it can maintain its complex behaviour indefinitely. Finding such a threshold is important; if the circuit has an error rate per elementary gate operation of no more than some threshold value $\tau$, then it is possible to construct reliable quantum circuits of arbitrary size. This should be contrasted with the fact that for the same error rate, the naive approach will only have reliable computation for timescales proportional to $1 / \tau$.

Why then don't we have large-scale quantum computers already? Unfortunately, the best known thresholds values for tolerable error rates are still very demanding, between $10^{-3}$ and $10^{-5}$, depending on the specifics of the architecture. Even the current best experiments suffer from much higher error rates than that. To bridge the gap between theory and practice, researchers work from both sides: experimentalists try to reduce the noise levels of their systems while theorists try to design better fault-tolerant schemes that allow higher error rates.

In light of the experimental challenges that we are facing, it would be useful to know what the best possible thresholds could be. Maybe the current threshold values are overly pessimistic, and thus $\mathrm{D}$-Wave might have a point when gambling

on the quantum adiabatic algorithm to ensure that computations survive the error rates of their implementation. But the work of Buhrman et al. ${ }^{2}$ shows that this kind of optimism has its limits: if the device is too noisy, any problem that it can solve can also be solved with the same efficiency using a standard laptop computer. To return to the analogy of fault-tolerant quantum computing as a self-sustaining ecosystem: for error rates that are above a threshold of approximately $45 \%$, the landscape resembles more the 'death zone' on the top of a mountain where no quantum computation can survive for a significant amount of time and all that remains is classical computation.

At the moment it is impossible to say if D-Wave's quantum computer is intrinsically equivalent to a classical computer or not. So until more is known about their error rates, caveat emptor is the least one can say.

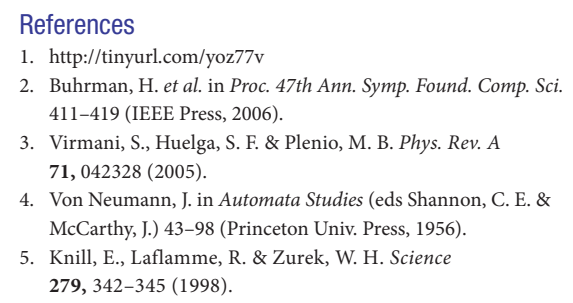

\title{
BIOPHYSICS
}

\section{The silence of the chromosomes}

In most mammals, males carry a pair of sex chromosomes designated 'XY', females carry 'XX' (pictured). Only one X chromosome per cell is needed for healthy development, and in females one of the pair of $\mathrm{X}$ chromosomes has to be silenced, that is, its gene expression switched off. Writing in Physical Review Letters, Mario Nicodemi and Antonella Prisco recognize this as a symmetry-breaking problem - how does a cell 'decide' which of the two chromosomes to silence? - and take a statistical-mechanics approach to solving it (Phys. Rev. Lett. 98, 108104; 2007).

Genetic studies have shown how the silencing itself works: each X chromosome includes a gene known as Xist, and RNA expressed from this gene shuts off further gene expression from the chromosome. Somehow, on one of the chromosomes but not the other, Xist is blocked and gene expression continues as normal. Nicodemi and Prisco consider how a blocking factor (perhaps an aggregate of proteins) could develop and then bind to one of the chromosomes, breaking the symmetry and protecting that chromosome from Xist.
In their model, particles (representing the likely components of the blocking factor) diffuse between points on a lattice that surrounds two parallel segments representing the $\mathrm{X}$ chromosomes. Interactions between particles and their nearest neighbours are described using an effective energy of either $E_{0}=2.4 k T$, equivalent to a weak hydrogen bond at room temperature, or $E_{0}=0$, which describes a random walk. Similarly, the binding energy at lattice sites associated with the chromosomes is set to $E_{\mathrm{X}}=2.4 k T$.

In the case of the random walk, Monte Carlo simulations show that there is no clustering of particles; but for $E_{0}=2.4 \mathrm{kT}$, there is. In a trade-off between energy and entropy, the clusters eventually build into a single complex around one of the chromosomes - breaking the symmetry of the system. The timescale of the process is determined by how close together the segments of the two chromosomes are: their observed proximity during silencing in biological systems could be necessary to be able to achieve the assembly of a blocking factor quickly enough.

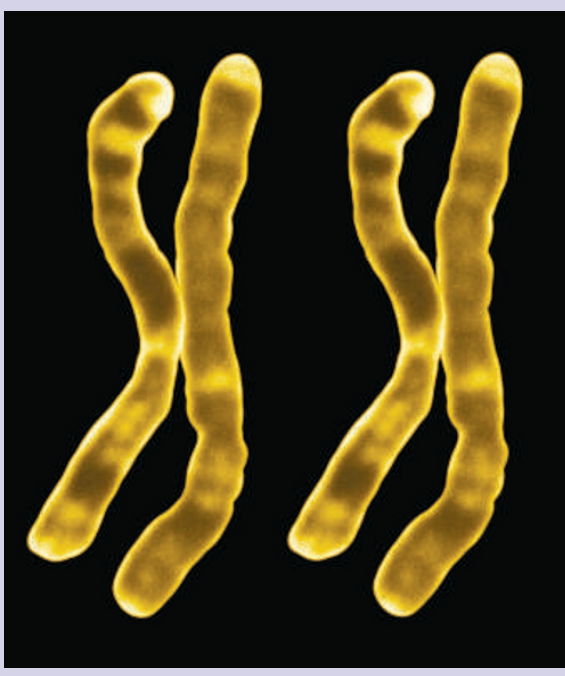

As the authors point out, $E_{\mathrm{X}}$ might not have the same value for each chromosome, due to copying errors during DNA replication. Then the symmetry is already broken, the chromosome with higher affinity will attract the blocking factor and save itself from silencing.

Alison Wright 